\title{
pandera: Statistical Data Validation of Pandas Dataframes
}

\author{
Niels Bantilan $\$$ \$* \\ https: //youtu.be/PxTLD-ueNd4
}

\begin{abstract}
-pandas is an essential tool in the data scientist's toolkit for modern data engineering, analysis, and modeling in the Python ecosystem. However, dataframes can often be difficult to reason about in terms of their data types and statistical properties as data is reshaped from its raw form to one that's ready for analysis. Here, I introduce pandera, an open source package that provides a flexible and expressive data validation API designed to make it easy for data wranglers to define dataframe schemas. These schemas execute logical and statistical assertions at runtime so that analysts can spend less time worrying about the correctness of their dataframes and more time obtaining insights and training models.
\end{abstract}

Index Terms—-data validation, data engineering

\section{Introduction}

pandas [WM10] has become an indispensible part of the data scientist's tool chain, providing a powerful interface for data processing and analysis for tabular data. In recent years numerous open source projects have emerged to enhance and complement the core pandas API in various ways. For instance, pyjanitor [EJMZBSZZS19] [pyj], pandas-ply [pdpa], and siuba [sba] are projects that provide alternative data manipulation interfaces inspired by the R ecosystem, pandas-profiling [pdpb] automatically creates data visualizations and statistics of dataframes, and dask [Roc15] provides parallelization capabilities for a variety of data structures, pandas dataframes among them.

This paper introduces a data validation tool called pandera, which provides an intuitive, flexible, and expressive API for validating pandas data structures at runtime. The problems that this library attempts to address are two-fold. The first is that dataframes can be difficult to reason about in terms of their contents and properties, especially when they undergo many steps of transformations in complex data processing pipelines. The second is that, even though ensuring data quality is critical in many contexts like scientific reporting, data analytics, and machine learning, the data validation process can produce considerable cognitive and software development overhead. Therefore, this tool focuses on making it as easy as possible to perform data validation in a variety of contexts and workflows in order to lower the barrier to explicitly defining and enforcing the assumptions about data.

* Corresponding author: niels.bantilan@ gmail.com

† Talkspace

$\S$ pyOpenSci

Copyright $@ 2020$ Niels Bantilan. This is an open-access article distributed under the terms of the Creative Commons Attribution License, which permits unrestricted use, distribution, and reproduction in any medium, provided the original author and source are credited.
In the following sections I outline the theoretical underpinnings and practical appications of data validation, describe in more detail the specific architecture and implementation of the pandera package, and compare and contrast it with similar tools in the Python and R ecosystems.

\section{Data Validation Definition}

Data validation is the process by which the data analyst decides whether or not a particular dataset fulfills certain properties that should hold true in order to be useful for some purpose, like modeling or visualization. In other words, data validation is a falsification process by which data is deemed valid with respect to a set of logical and statistical assumptions [VdLDJ18]. These assumptions are typically formed by interacting with the data, where the analyst may bring to bear some prior domain knowledge pertaining to the dataset and data manipulation task at hand. Notably, even with prior knowledge, exploratory data analysis is an essential part of the workflow that is part of the data wrangling process.

More formally, we can define data validation in its most simple form as a function:

$$
v(x) \rightarrow\{\text { True }, \text { False }\}
$$

Where $v$ is the validation function, $x$ is the data to validate, and the output is a boolean value. As [vdLdJ19] points out, the validation function $v$ must be a surjective (onto) function that covers the function's entire range in order to be meaningful. To see why, consider a validation function that always returns True or always returns False. Such a function cannot falsify any instantiation of the dataset $x$ and therefore fails to provide any meaningful information about the validity of any dataset ${ }^{1}$. Although the above formulation covers a wide variety of data structures, this paper will focus on tabular data.

\section{Types of Validation Rules}

[vdLdJ19] distinguishes between technical validation rules and domain-specific validation rules. Technical validation rules describe the variables, data types, and meta-properties of what constitutes a valid or invalid data structure, such as uniqueness and nullability. On the other hand, domain-specific validation rules

1. There are nuances around how to formulate the domain of the function $v$. For a more comprehensive formal treatment of data validation, refer to [vdLdJ19] and [VdLDJ18] 
describe properties of the data that are specific to the particular topic under study. For example, a census dataset might contain age, income, education, and job_category columns that are encoded in specific ways depending on the way the census was conducted. Reasonable validation rules might be:

- The age and income variables must be positive integers.

- The age variable must be below $122^{2}$.

- Records where age is below the legal working age should have NA values in the income field.

- education is an ordinal variable that must be a member of the ordered set nnone, high school, undergraduate, graduate\}.

- job_category is an unordered categorical variable that must be a member of the set \{professional, manegerial, service, clerical, agricultural, technical\}.

We can also reason about validation rules in terms of the statistical and distributional properties of the data under validation. We can think about at least two flavors of statistical validation rules: deterministic, and probabilistic. Probabilistic checks explicitly express uncertainty about the statistical property under test and encode notions of stochasticity and randomness. Conversely, deterministic checks express assertions about the data based on logical rules or functional dependencies that do not explicitly incorporate any assumptions about randomness into the validation function.

Often times we can express statistical properties about data using deterministic or probabilistic checks. For example, "the mean age among the graduate sample tends to be higher than that of the undergraduate sample in the surveyed population" can be verified deterministically by simply computing the means of the two samples and applying the logical rule mean $\left(\right.$ age $\left._{\text {graduate }}\right)>$ mean $\left(\right.$ age $\left._{\text {undergraduate }}\right)$. A probabilistic version of this check would be to perform a hypothesis test, like a t-test with a pre-defined alpha value. Most probabilistic checks can be reduced to deterministic checks, for instance by simply evaluating the truth/falseness of a validation rule using the test statistic that results from the hypothesis test and ignoring the p-value. Doing this simplifies the validation rule but trades off simplicity for being unable to express uncertainty and statistical significance. Other examples of such probabilistic checks might be:

- The income variable is positively correlated with the education variable.

- income is negatively correlated with the dummy variable job_category_service, which is a variable derived from the job_category column.

\section{Data Validation in Practice}

Data validation is part of a larger workflow that involves processing raw data to produce of some sort of statistical artifact like a model, visualization, or report. In principle, if one can write perfect, bug-free code that parses, cleans, and reshapes the data to produce these artifacts, data validation would not be necessary. In practice, however, data validation is critical for preventing the silent passing of an insidious class of data integrity error, which

2. The age of the oldest person: https://en.wikipedia.org/wiki/List_of_the verified_oldest_people

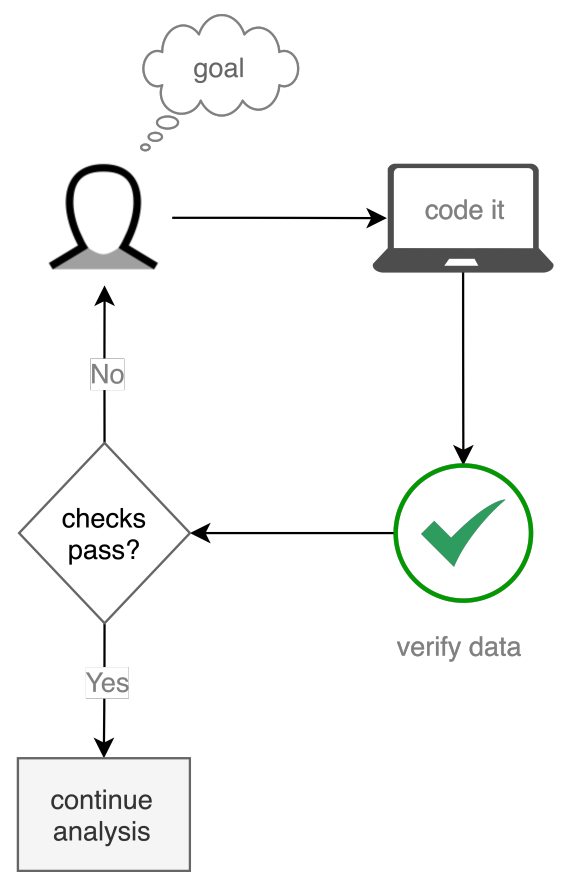

Fig. 1: Data validation as an iterative software development process.

is otherwise difficult to catch without explicitly making assertions at runtime. These errors could lead to misleading visualizations, incorrect statistical inferences, and unexpected behavior in machine learning models. Explicit data validation becomes even more important when the end product artifacts inform business decisions, support scientific findings, or generate predictions about people or things in the real world.

Consider the process of constructing a dataset for training a machine learning model. In this context, the act of data validation is an iterative loop that begins with the analyst's objective and a mental model of what the data should "look" like. She then writes code to produce the dataset of interest, simultaneously inspecting, summarizing, and visualizing the data in an exploratory fashion, which in turn enables her to build some intuition and domain knowledge about the dataset.

She can then codify this intuition as a set of assumptions, implemented as a validation function, which can be called against the data to ensure that they adhere to those assumptions. If the validation function evaluates to False against the data during development time, the analyst must decide whether to refactor the processing logic to fulfill the validation rules or modify the rules themselves $^{3}$.

In addition to enforcing correctness at runtime, the resulting validation function also documents the current state of assumptions about the dataset for the benefit of future readers or maintainers of the codebase.

The role of the analyst, therefore, is to encode assumptions about data as a validation function and maintain that function as new datasets pass through the processing pipeline and the

3. In the latter scenario, the degenerate case is to remove the validation function altogether, which exposes the program to the risks associated with silently passing data integrity errors. Practically, it is up to the analyst to determine an appropriate level of strictness that catches cases that would produce invalid outputs. 
definition of valid data evolves over time. One thing to note here is that using version control software like git [git] would keep track of the changes of the validation rules, enabling maintainers or readers of the codebase to inspect the evolution of the contract that the data must fulfill to be considered valid.

\section{Design Principles}

pandera is a flexible and expressive API for pandas data validation, where the goal is to provide a data engineering tool that (i) helps pandas users reason about what clean data means for their particular data processing task and (ii) enforce those assumptions at run-time. The following are the principles that have thus far guided the development of this project:

- Expressing validation rules should feel familiar to pandas users.

- Data validation should be compatible with the different workflows and tools in the data science toolbelt without a lot of setup or configuration.

- Defining custom validation rules should be easy.

- The validation interface should make the debugging process easier.

- Integration with existing code should be as seamless as possible.

These principles articulate the use cases that I had when surveying the Python ecosystem for pandas data validation tools.

\section{Architecture}

pandera helps users define schemas as contracts that a pandas dataframe must fulfill. This contract specifies deterministic and statistical properties that must hold true to be considered valid with respect to a particular analysis. Since pandera is primarily a data engineering tool, the validation function defined in Equation (1) needs to be slightly refactored:

$$
s(v, x) \rightarrow \begin{cases}\mathrm{x}, & \text { if } v(x)=\text { true } \\ \text { error, } & \text { otherwise }\end{cases}
$$

Where $s$ is a schema function that takes the validation function from Equation (1) and some data as input and returns the data itself if it is valid and an error otherwise. In pandera, the error is implemented as a SchemaError exception that contains the invalid data as well as a pandas dataframe of failure cases that contains the index and failure case values that caused the exception.

The primary rationale for extending validation functions in this way is that it enables users to compose schemas with data processing functions, for example, $s \circ f(x)$ is a composite function that first applies a data processing function $f$ to the dataset $x$ and then validates the output with the schema $s$. Another possible composite function, $f \circ s(x)$, applies the validation function to $x$ before applying the $f$, effectively guaranteeing that inputs to $f$ fulfill the contract enforced by $s$.

This formulation of data validation facilitates the interleaving of data processing and validation code in a flexible manner, allowing the user to decide the critical points of failure in a pipeline where data validation would make it more robust to abherrant data values.

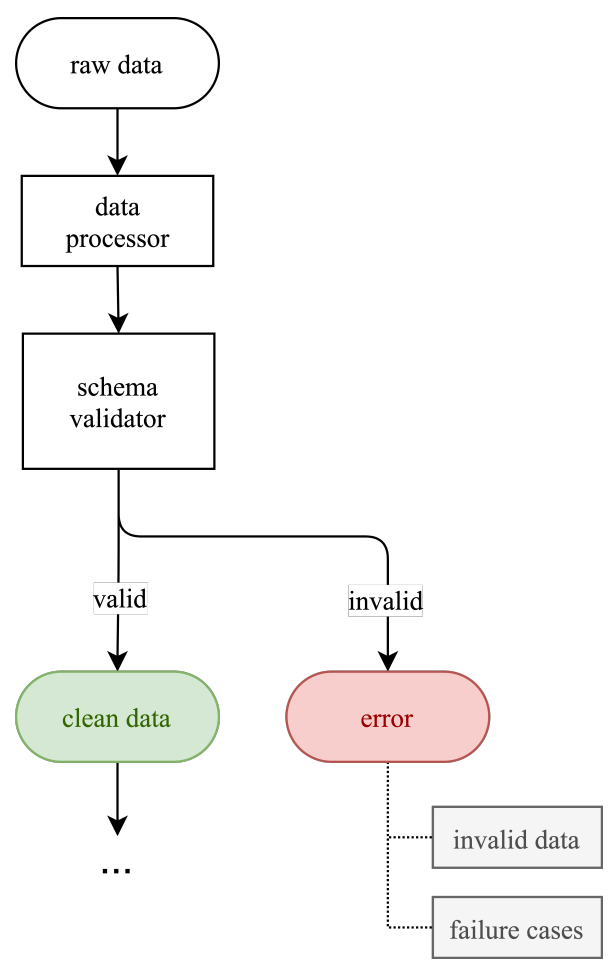

Fig. 2: High-level architecture of pandera. In the simplest case, raw data passes through a data processor, is checked by a schema validator, and flows through to the next stage of the analysis pipeline if the validation checks pass, otherwise an error is raised.

\section{Core Features}

\section{DataFrameSchemas as Contracts}

The main concepts of pandera are schemas, schema components, and checks. Schemas are callable objects that are initialized with validation rules. When called with compatible data as an input argument, a schema object returns the data itself if the validation checks pass and raises a SchemaError when they fail. Schema components behave in the same way as schemas but are primarily used to specify validation rules for specific parts of a pandas object, e.g. columns in a dataframe. Finally, checks allow the users to express validation rules in relation to the type of data that the schema or schema component are able to validate.

More specifically, the central objects in pandera are the DataFrameSchema, Column, and Check. Together, these objects enable users to express schemas upfront as contracts of logically grouped sets of validation rules that operate on pandas dataframes. For example, consider a simple dataset containing data about people, where each row is a person and each column is an attribute about that person:

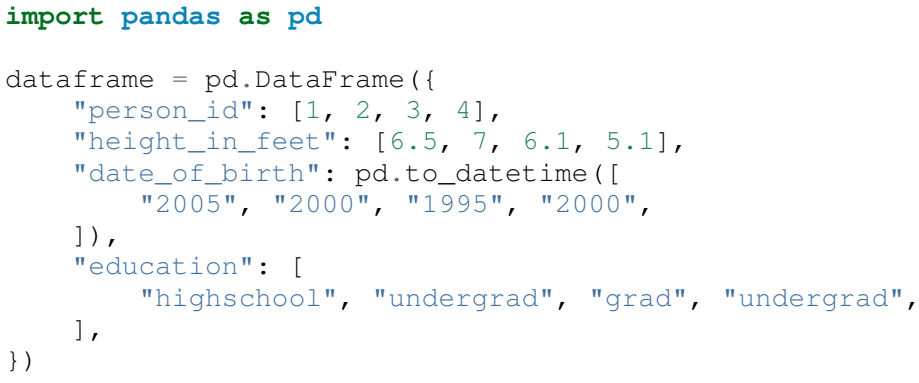

We can see from inspecting the column names and data values that we can bring some domain knowledge about the world to express 
our assumptions about what are considered valid data.

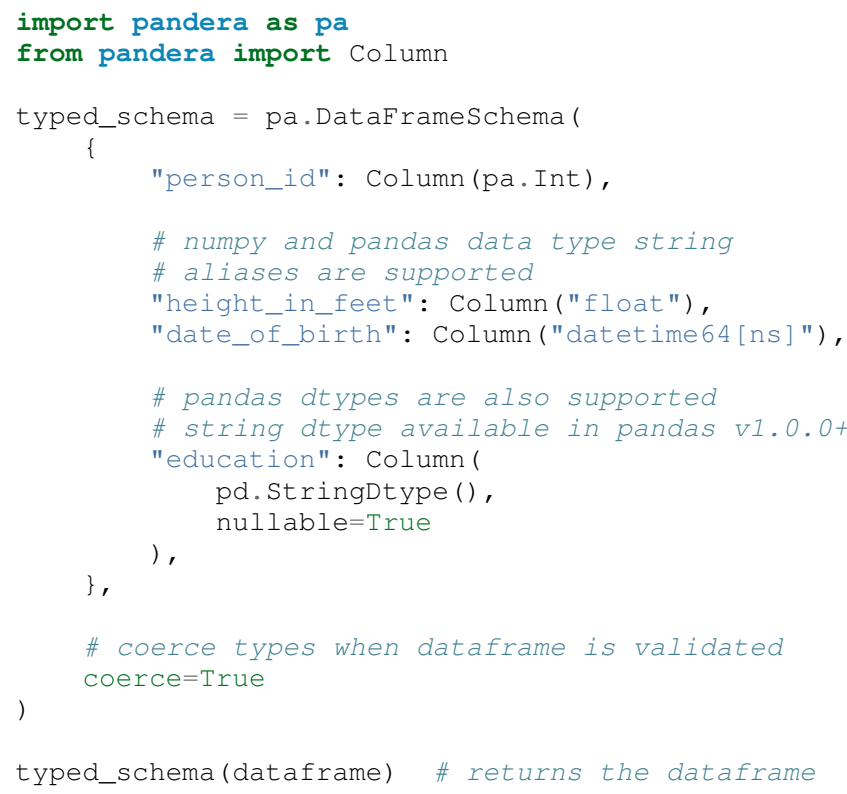

\section{Validation Checks}

The typed_schema above simply expresses the columns that are expected to be present in a valid dataframe and their associated data types. While this is useful, users can go further by making assertions about the data values that populate those columns:

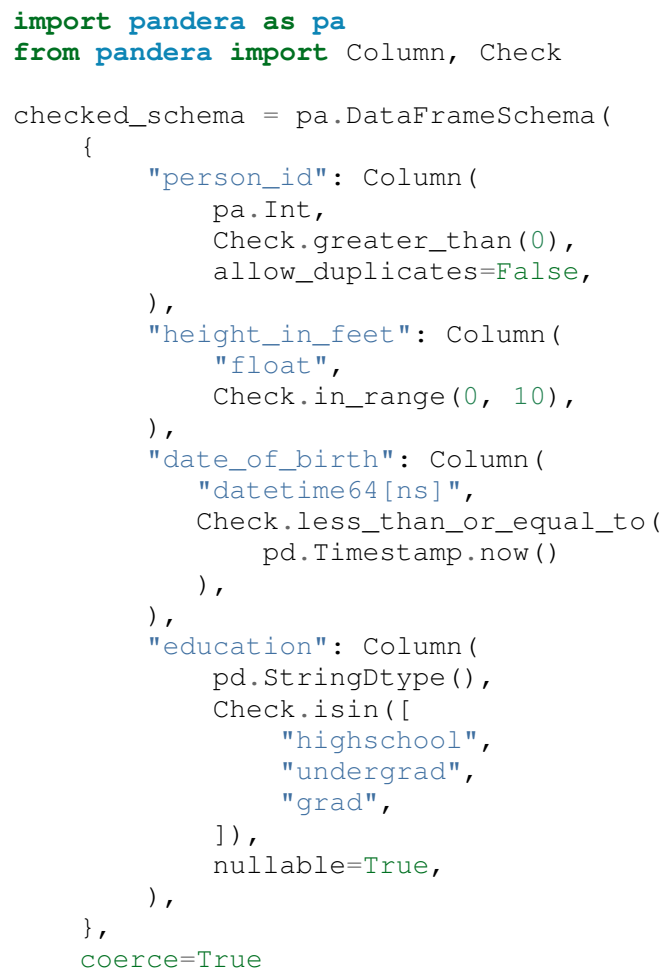

The schema definition above establishes the following properties about the data:

- the person_id column is a positive integer, which is a common way of encoding unique identifiers in a dataset. By setting allow_duplicates to False, the schema indicates that this column is a unique identifier in this dataset.
- height_in_feet is a positive float whose maximum value is 10 feet, which is a reasonable assumption for the maximum height of human beings.

- date_of_birth cannot be a date in the future.

- education can take on the acceptable values in the set \{"highschool", "undergrad", "grad"\}. Supposing that these data were collected in an online form where the education field input was optional, it would be appropriate to set nullable to True (this argument is False by default).

\section{Error Reporting and Debugging}

If a dataframe passed into the schema callable object does not pass the validation checks, pandera provides an informative error message:

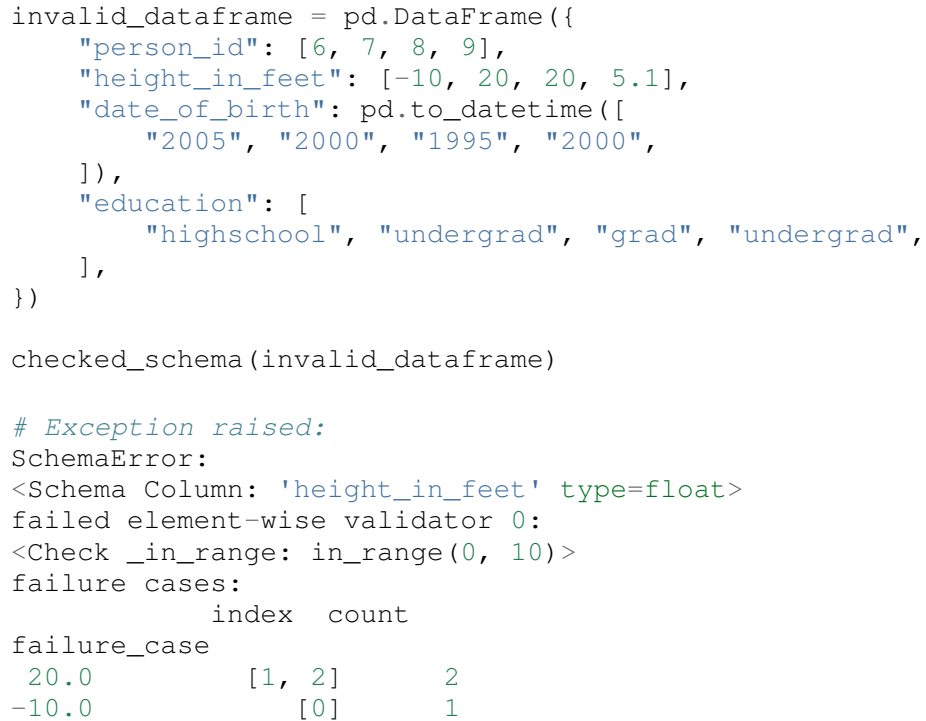

The causes of the SchemaError are displayed as a dataframe where the failure_case index is the particular data value that failed the Check. in_range validation rule, the index column contains a list of index locations in the invalidated dataframe of the offending data values, and the count column summarizes the number of failure cases of that particular data value.

For finer-grained debugging, the analyst can catch the exception using the try...except pattern to access the data and failure cases as attributes in the SchemaError object:

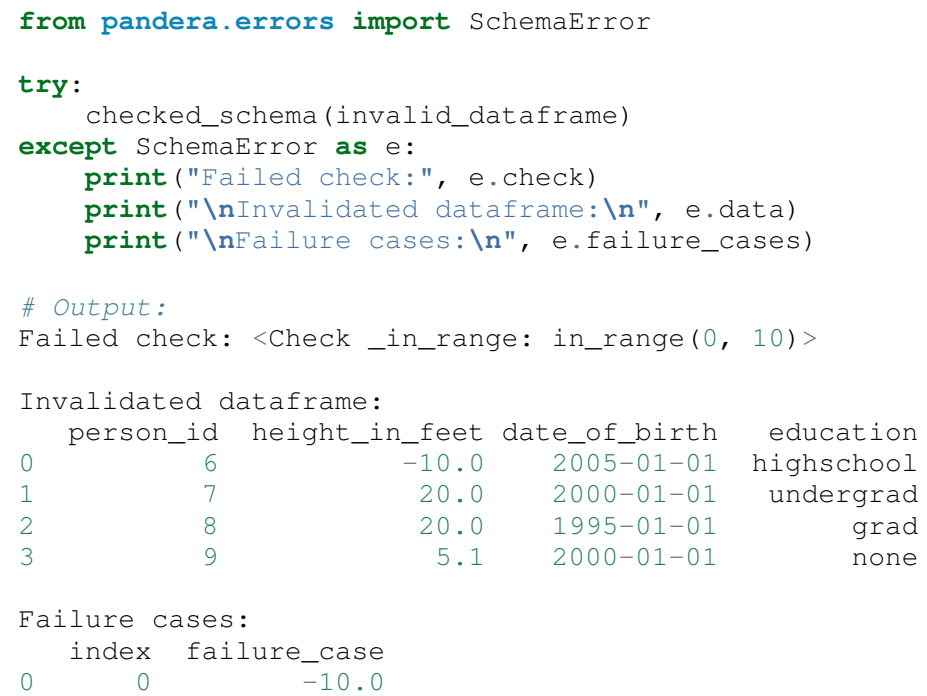




$\begin{array}{lll}1 & 1 & 20.0 \\ 2 & 2 & 20.0\end{array}$

In this way, users can easily access and inspect the invalid dataframe and failure cases, which is especially useful in the context of long method chains of data transformations:

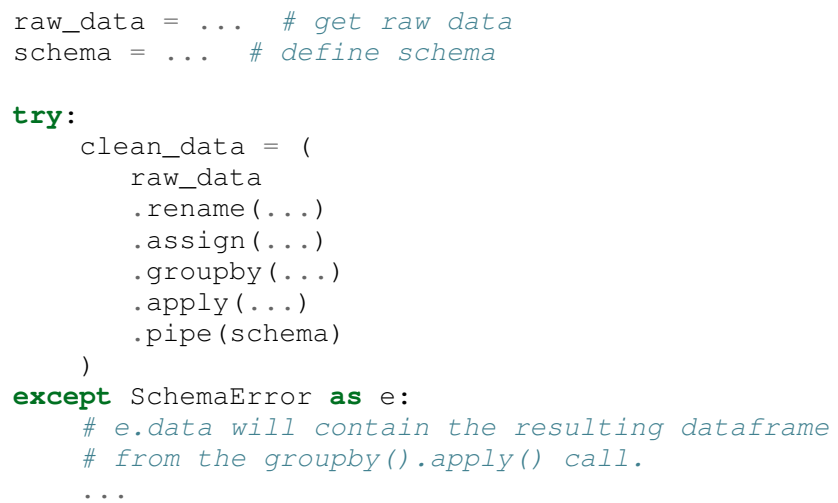

\section{Pipeline Integration}

There are several ways to interleave pandera validation code with data processing code. As shown in the example above, one can use a schema by simply using it as a callable. Users can also sandwich data preprocessing code between two schemas; one schema that ensures the raw data fulfills certain assumptions, and another that ensures the processed data fulfills another set of assumptions that arise as a consequence of the data processing. The following code provides a toy example of this pattern:

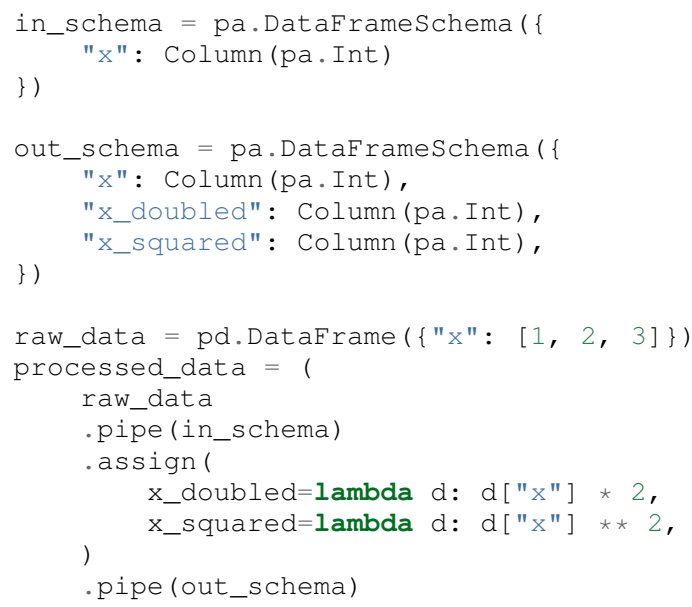

For more complex pipelines that handle multiple steps of data transformations with functions, pandera provides a decorator utility for validating the inputs and outputs of functions. The above example can be refactored into:

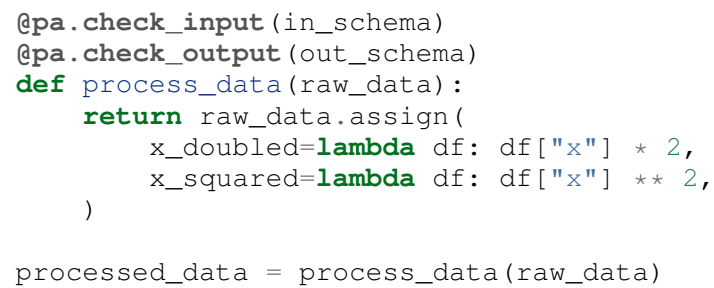

\section{Custom Validation Rules}

The Check class defines a suite of built-in methods for common operations, but expressing custom validation rules are easy. In the simplest case, a custom column check can be defined simply by passing a function into the Check constructor. This function needs to take as input a pandas Series and output either a boolean or a boolean Series, like so:

Column ( checks=Check (lambda s: $\operatorname{s.between}(0,1))$ )

The element_wise keyword argument changes the expected function signature to a single element in the column, for example, a logically equivalent implementation of the above validation rule would be:

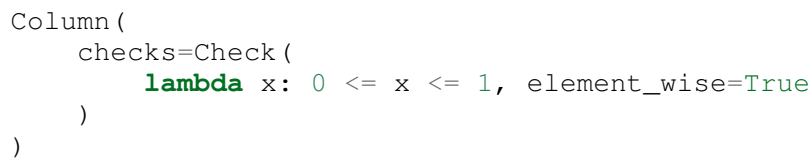

Check objects can also be used in the context of a DataFrameSchema, in which case the function argument should take as input a pandas DataFrame and output a boolean, a boolean Series, or a boolean DataFrame.

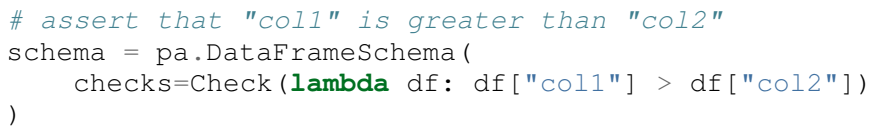

Currently, in the case that the check function returns a boolean Series or DataFrame, all of the elements must be True in order for the validation check to pass.

\section{Advanced Features}

\section{Hypothesis Testing}

To provide a feature-complete data validation tool for data scientists, pandera subclasses the Check class to define the Hypothesis class for the purpose of expressing statistical hypothesis tests. To illustrate one of the use cases for this feature, consider a toy scientific study where a control group receives a placebo and a treatment group receives a drug that is hypothesized to improve physical endurance. The participants in this study then run on a treadmill (set at the same speed) for as long as they can, and running durations are collected for each individual.

Even before collecting the data, we can define a schema that expresses our expectations about a positive result:

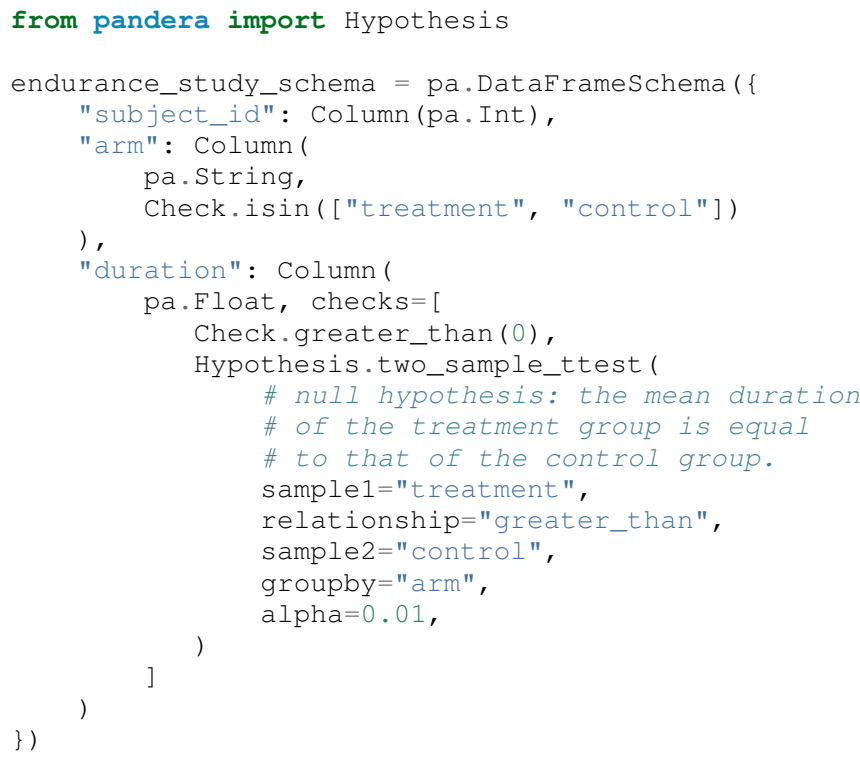

Once the dataset is collected for this study, we can then pass it through the schema to validate the hypothesis that the group 
receiving the drug increases physical endurance, as measured by running duration.

As of version 0.4 .0 , the suite of built-in hypotheses is limited to the two_sample_ttest and one_sample_ttest, but creating custom hypotheses is straight-forward. To illustrate this, another common hypothesis test might be to check if a sample is normally distributed. Using the scipy.stats.normaltest function, one can write:

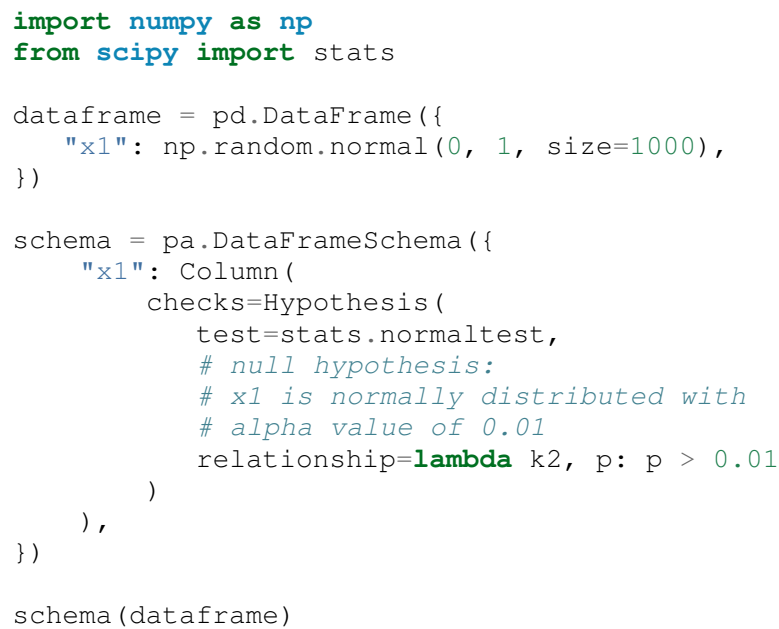

\section{Conditional Validation Rules}

If we want to validate the values of one column conditioned on another, we can provide the other column name in the groupby argument. This changes the expected Check function signature to expect an input dictionary where the keys are discrete group levels in the conditional column and values are pandas Series objects containing subsets of the column of interest. Returning to the endurance study example, we could simply assert that the mean running duration of the treatment group is greater than that of the control group without assessing statistical significance:

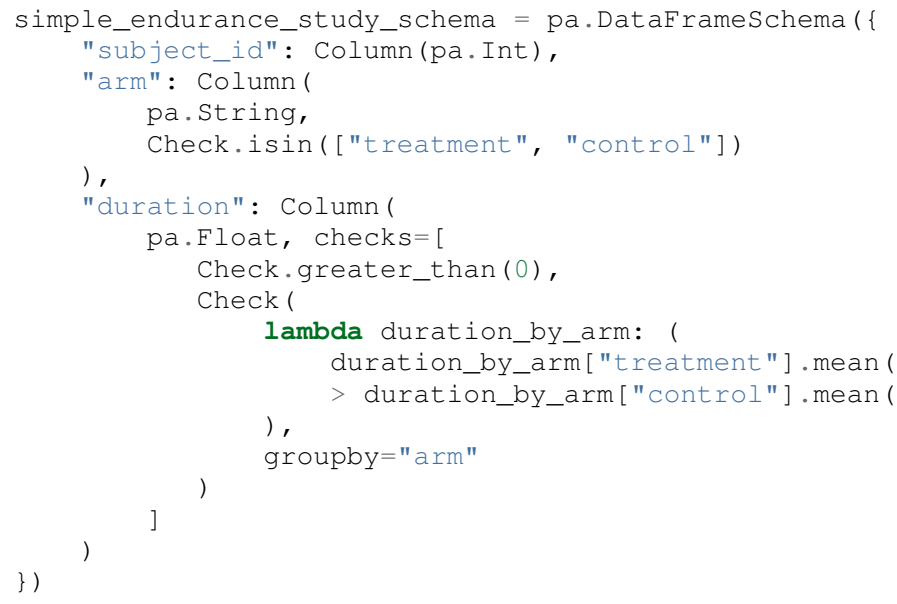

Functional dependencies are a type of conditional validation rule that expresses a constraint between two sets of variables in a relational data model [Arm74] $\left[\mathrm{BFG}^{+} 07\right]$. For example, consider a dataset of biological species where each row is a species and each column is a classification in the classic hierarchy of kingdom $->$ phylum $->$ class $->$ order ... -> species. We can assert that "if two species are in the same phylum, then they must be in the same kingdom":

species_schema $=$ pa.DataFrameSchema $(\{$

"phylum": Column(pa.String),

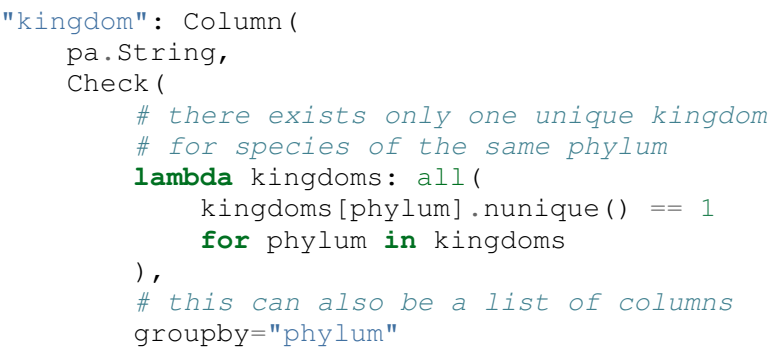

However, in order to make the assertion "if two species are in the same order, then they must be in the same class and phylum", we have to use dataframe-level checks since the above pattern can only operate on values of a single column grouped by one or more columns.

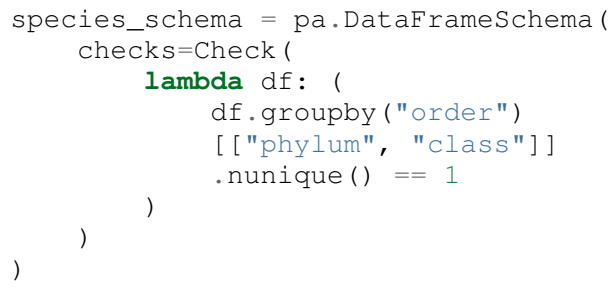

\section{Use Case Vignettes}

This section showcases the types of use cases that pandera is designed to address via hypothetical vignettes that nevertheless illustrate how pandera can be beneficial with respect to the maintainability and reproducibility of analysis/model pipeline code. These vignettes are based on my experience using this library in research and production contexts.

\section{Catching Type Errors Early}

Consider a dataset of records with the fields age, occupation, and income, where we would like to predict income as a function of the other variables. A common type error that arises, especially when processing unnormalized data or flat files, is the presence of values that violate our expectations based on domain knowledge about the world:

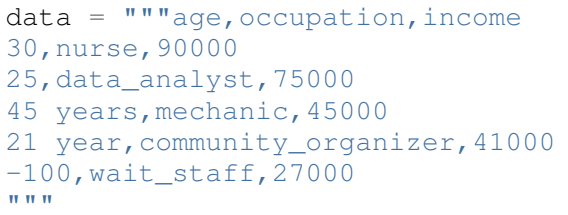

In the above example, the age variable needs to be cleaned so that its values are positive integers, treating negative values as null.

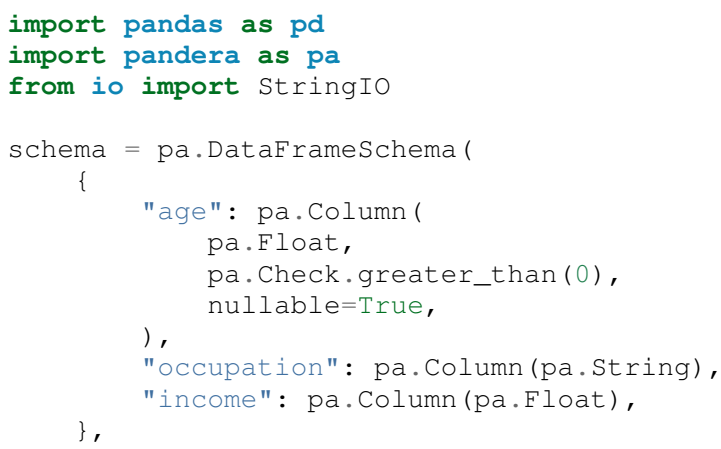


coerce $=$ True

pd.read_csv (StringIo(data)).pipe (schema)

\# ValueError:

\# invalid literal for int() with base 10: '45 years'

Defining a data cleaning function would be standard practice, but here we can augment this function with guard-rails that would catch age values that cannot be cast into a float type and convert negative values to nulls.

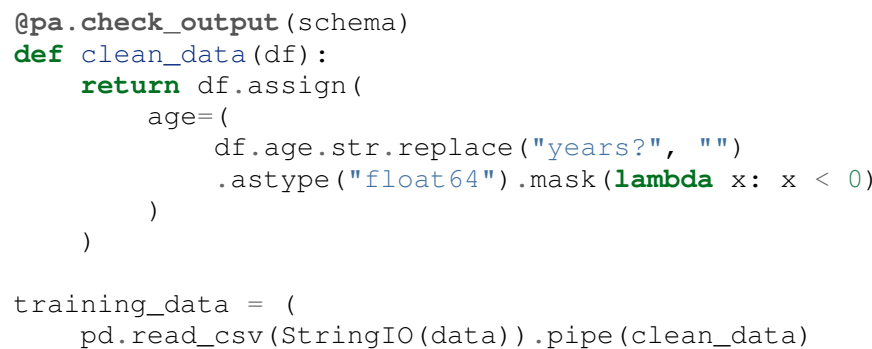

The implementation of clean_data now needs to adhere to the schema defined above. Supposing that the data source is refreshed periodically from some raw data feed, additional records with age values like 22 years and 7 months would be caught early in the data cleaning portion of the pipeline, and the implementation within clean_data would have to be refactored to normalize these kinds of more complicated values.

Though this may appear to be a trivial problem, validation rules on unstructured data types like text benefit greatly from even simple validation rules, like checking that values are nonempty strings and contain at least a minimum number of tokens, before sending the text through a tokenizer to produce a numerical vector representation of the text. Without these validation checks, these kinds of data integrity errors would pass silently through the pipeline, only to be unearthed after a potentially expensive model training run.

\section{Reusable Schema Definitions}

In contexts where the components of an ML pipeline are handled by different services, we can reuse and modify schemas for the purposes of model training and prediction. Since schemas are just python objects, schema definition code can be placed in a module e.g. schemas.py, which can then be imported by the model training and prediction modules.

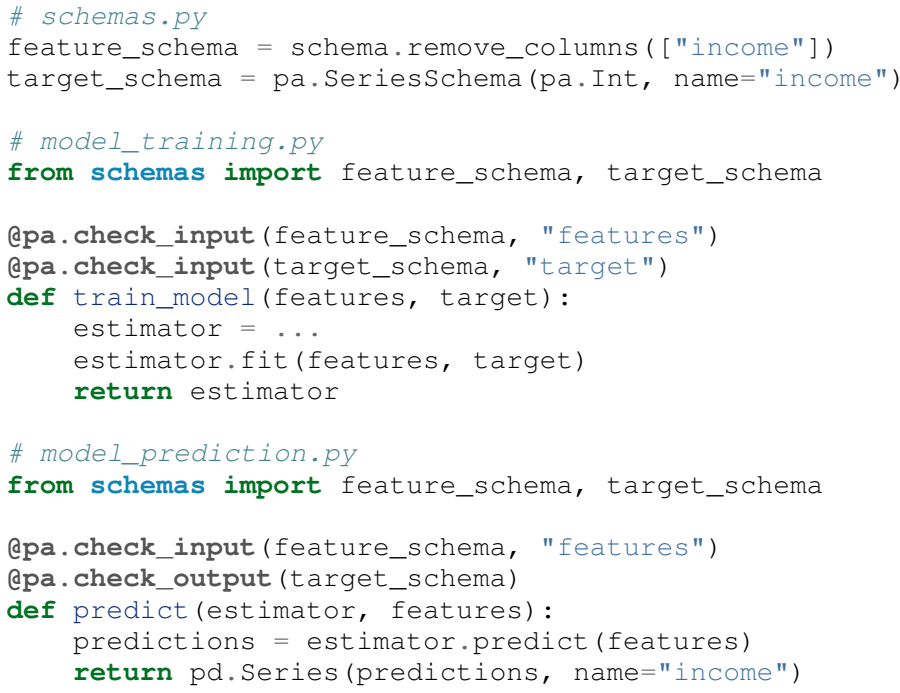

\section{Unit Testing Statistically-Typed Functions}

Once functions are decorated with check_input or check_output, we can write unit tests for them by generating synthetic data that produces the expected results. For example, here is a test example using pytest [pyt]:

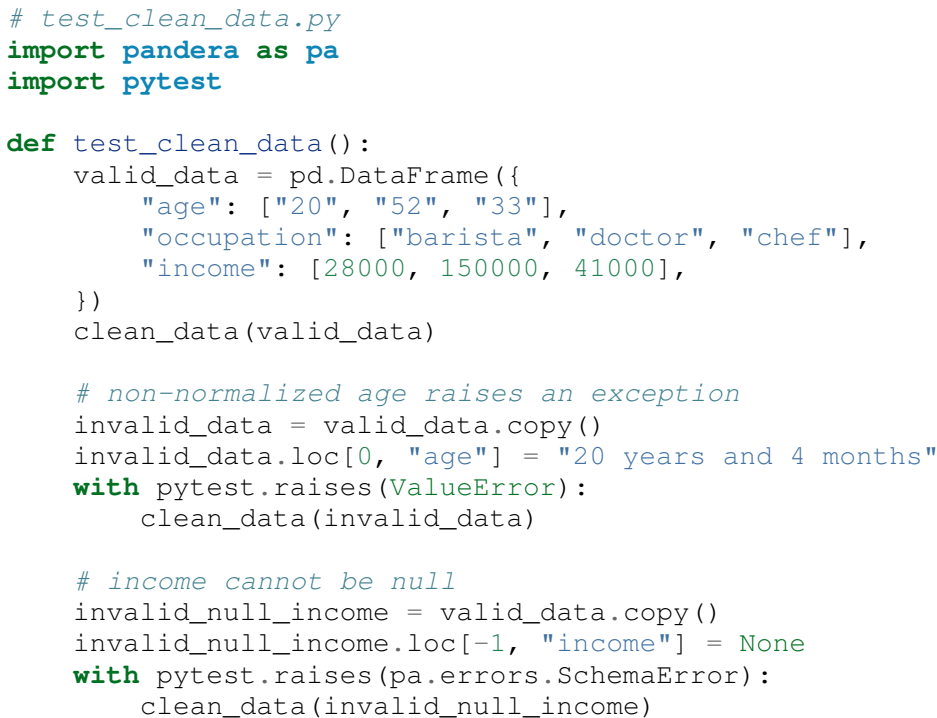

This last use case would be further enhanced by property-based testing libraries like hypothes is [MHDC19] [MHDpb $\left.{ }^{+} 20\right]$ that could be used to generate synthetic data against which to test schema-decorated functions.

\section{Documentation}

Documentation for pandera is hosted on ReadTheDocs, where tutorials on core and experimental features are available, in addition to full API documentation.

\section{Limitations}

The most notable limitation of pandera is the computational cost of running validation checks at runtime. This limitation applies to any data validation code, which trades off increased run-time for type safety and data integrity. The project currently uses airspeed-velocity [asv] for a few basic run-time and memory usage benchmarks, but more extensive performance profiling is warranted to give users a better sense of this trade-off. The other trade-off to consider is the additional development time associated with defining robust and meaningful schemas versus the time spent debugging silent data integrity issues, which is particularly costly in areas like machine learning where model debugging occurs after training a model.

A related limitation is that type-checking schemas are practical for large datasets (e.g. datasets that do not fit onto disk in a modern laptop), but validation checks that verify statistics on one or more columns can become expensive. For this reason, the default Check function signature is expected to be a Series in order to encourage users to use the optimized pandas. Series methods. In theory, pandera schemas can be coupled with parallelization tools like dask [Roc15] to perform data validation in these settings.

Two other limitations of the current state of the package are that:

- The built-in Hypothesis methods are currently limited in scope, and implementing wrapper methods to 
the scipy implementations of commonly used distributional tests (e.g. normality test, chi-squared test, and KLdivergence) would encourage the use of hypothesis tests in schemas.

- Expressing functional dependencies is currently inelegant and would benefit from a higher-level abstraction to improve usability.

\section{Roadmap}

The pandera project started as a naive excursion into seeing whether pandas dataframes could be statically typed, as gradual typing is becoming adopted by the Python community since the typing module was introduced in Python 3.5. The project evolved into a tool that emphasizes the verification of the statistical properties of data, which requires run-time validation.

The direction of this project has been driven, in large part, by its contributors, and will continue to be via feature requests on the github repo. There are a number of experimental features that are currently available in version $0.4 .0+$ that aim to speed up the iteration loop of defining schemas at development time through interactive analysis:

- schema inference: the pandera.infer_schema function takes as input a dataframe and outputs an automatically generated draft schema that the user can iterate on.

- yaml/module serialization: this feature enables the user to write schemas (inferred or otherwise) to a yaml file or python script, which are editable artifacts to iterate on.

Additionally, a few feature proposals would benefit from discussion and feedback from the wider scientific computing and data science community:

- Synthetic data generation based on schema definitions [issue 200].

- Domain-specific schemas, types, and checks, e.g. for the machine learning use case, provide first-class support for validation checks between target and feature variables [issue 179].

- Expressing a tolerance level for the proportion of values that fail a validation Check [issue 183].

There are several ways to contribute for interested readers:

- Improving documentation by adding examples, fixing bugs, or clarifying the the writing.

- Feature requests: e.g. requests for additional built-in Check and Hypotheses methods.

- Submit new issues or pull requests for existing issues.

\section{Related Tools}

This project was inspired by the schema and pandas_schema Python packages and the validate $\mathrm{R}$ package [vdLdJ19]. Initially when assessing the Python landscape for pandas-centric data validation tools, I found that they did not match my use cases because they (a) often resulted in verbose and over-specified validation rulesets, (b) introduced many new library-specific concepts and configuration steps, (c) lacked documentation of core functionality and usage patterns, and/or (d) are no longer maintained.

Here is my assessment of data validation tools that are currently being maintained in the Python ecosystem:
- great_expectations [ge]: this is a mature, batteriesincluded data validation library centered around the concept of expectations. It provides a UI to manage validation rules and supports integrations with many database systems and data manipulation tools. This framework extends the pandas.DataFrame class to include validation methods prefixed with expect_* and a suite of built-in rules for common use cases. Defining custom validation rules involves subclassing the PandasDataset class and defining specially-decorated methods with function signatures that adhere to library-specific standards.

- schema [sch]: a light-weight data validator for generic Python data structures. This package and pandera share the schema interface where the schema object returns the data itself if valid and raises an Exception otherwise. However, this library does not provide additional functionality for pandas data structures.

- pandas_schema [ps]: a pandas data validation library with a comprehensive suite of built-in validators. This package was the inspiration for the schema component design where a Column object specifies properties of a dataframe column, albeit the specific implementations are considerably different. It provides built-in validators and supports defining custom validation rules. Unlike pandera which outputs the validated data, the output of validating a dataframe with pandas_schema is an iterable of errors that are intended to be inspected via print statements.

The key features that differentiate pandera from similar packages in the Python ecosystem are the following:

- check_input and check_output function decorators enable seamless integration with existing data processing/analysis code.

- Check validation rules are designed primarily for customizability, with built-in methods as a convenience for common validation rules.

- Hypothes is validation rules provide a tidy-first $\left[\mathrm{W}^{+} 14\right]$ interface for hypothesis testing.

- Ease of debugging, as SchemaErrors contain the invalidated data as well as a tidy dataframe of the failure cases with their corresponding column/index locations.

- Schema inference and serialization capabilities enable the creation of draft schemas that users can iterate on and refine.

- Clear and comprehensive documentation on core and advanced features.

\section{Conclusion}

This paper introduces the pandera package as a way of expressing assumptions about data and falsifying those assumptions at run time. This tool is geared toward helping data engineers and data scientists during the software development process, enabling them to make their data proprocessing workflows more readable, robust, and maintainable.

\section{Acknowledgements}

I would like to acknowledge the pyOpenSci community for their support and the pandera contributors who have made significant improvements and enhancements to the project. 


\section{REFERENCES}

[Arm74] William Ward Armstrong. Dependency structures of data base relationships. In IFIP congress, volume 74, pages 580-583. Geneva, Switzerland, 1974.

[asv] airspeed velocity. Accessed: 29 May 2020. URL: https: //github.com/airspeed-velocity/asv.

$\left[\mathrm{BFG}^{+}\right.$07] Philip Bohannon, Wenfei Fan, Floris Geerts, Xibei Jia, and Anastasios Kementsietsidis. Conditional functional dependencies for data cleaning. In 2007 IEEE 23rd international conference on data engineering, pages 746-755. IEEE, 2007.

[EJMZBSZZS19] Eric J. Ma, Zachary Barry, Sam Zuckerman, and Zachary Sailer. pyjanitor: A Cleaner API for Cleaning Data. In Chris Calloway, David Lippa, Dillon Niederhut, and David Shupe, editors, Proceedings of the 18th Python in Science Conference, pages 50 - 53, 2019. doi:10.25080/ Majora-7ddc1dd1-007.

[ge] Great expectations: Always know what to expect from your data. Accessed: 29 May 2020. URL: https://github.com/ great-expectations/great_expectations.

[git] Git. Accessed: 29 May 2020. URL: https://git-scm.com.

[MHDC19] David MacIver, Zac Hatfield-Dodds, and Many Contributors. Hypothesis: A new approach to property-based testing. Journal of Open Source Software, 4(43):1891, 11 2019. URL: http://dx.doi.org/10.21105/joss.01891, doi : $10.21105 /$ joss. 01891.

$\left[\mathrm{MHDpb}^{+}{ }^{20]}\right.$ David R. MacIver, Zac Hatfield-Dodds, pyup.io bot, Alex Chan, Stuart Cook, Ryan Soklaski, David Chudzicki, jwg4, Alex Willmer, Tyler, Kyle Reeve, Grigorios Giannakopoulos, mulkieran, Emmanuel Leblond, Christopher Armstrong, Tyler Gibbons, Jeremy Thurgood, Paul Stiverson, SuperStormer, Alexander Shorin, David Mascharka, Peter C Kroon, Anne Archibald, Tom Prince, Mathieu PATUREL, dwest netflix, Tom McDermott, rdturnermtl, Graham Williamson, and Cory Benfield. HypothesisWorks/hypothesis: Hypothesis for Python - version 5.16.0, May 2020. URL: https://doi.org/10.5281/zenodo.3859851, doi:10.5281/zenodo.3859851.

[pdpa] pandas-ply. Accessed: 6 June 2020. URL: https://github. com/coursera/pandas-ply.

[pdpb] pandas-profiling. Accessed: 6 June 2020. URL: https:// github.com/pandas-profiling/pandas-profiling.

[ps] Pandasschema. Accessed: 29 May 2020. URL: https:// github.com/TMiguelT/PandasSchema.

[pyj] pyjanitor. Accessed: 6 June 2020. URL: https://github.com/ ericmj1/pyjanitor.

[pyt] pytest. Accessed: 29 June 2020. URL: https://github.com/ pytest-dev/pytest.

[Roc15] Matthew Rocklin. Dask: Parallel computation with blocked algorithms and task scheduling. In Proceedings of the 14th python in science conference, number 130-136. Citeseer, 2015.

[sba] siuba. Accessed: 6 June 2020. URL: https://github.com/ machow/siuba.

[sch] Schema: Schema validation just got pythonic. Accessed: 29 May 2020. URL: https://github.com/keleshev/schema.

[VdLDJ18] Mark Van der Loo and Edwin De Jonge. Statistical data cleaning with applications in $R$. Wiley Online Library, 2018.

[vdLdJ19] Mark PJ van der Loo and Edwin de Jonge. Data validation infrastructure for r. arXiv preprint arXiv:1912.09759, 2019.

$\begin{array}{ll}{\left[\mathrm{W}^{+} 14\right]} & \text { Hadley Wickham et al. Tidy data. Journal of Statistical } \\ & \text { Software, 59(10):1-23, 2014. } \\ \text { [WM10] } & \text { Wes McKinney. Data Structures for Statistical Computing }\end{array}$

$\begin{array}{ll}{\left[\mathrm{W}^{+} 14\right]} & \text { Hadley Wickham et al. Tidy data. Journal of Statistical } \\ & \text { Software, 59(10):1-23, 2014. } \\ \text { [WM10] } & \text { Wes McKinney. Data Structures for Statistical Computing }\end{array}$ in Python. In Stéfan van der Walt and Jarrod Millman, editors, Proceedings of the 9th Python in Science Conference, pages 56-61,2010. doi:10.25080/Majora92bf1922-00a. 